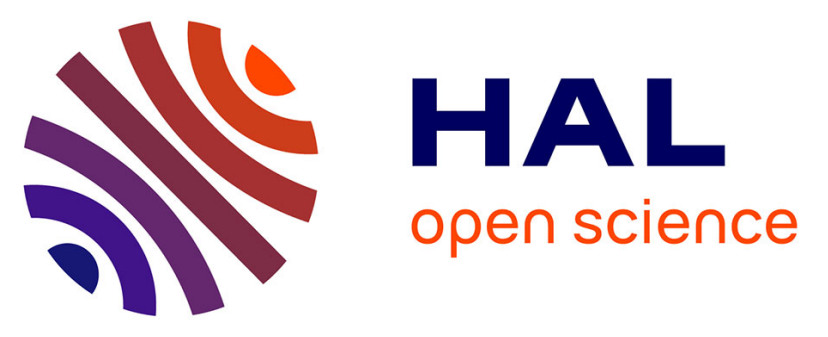

\title{
Implicit active contours for ultrasound images segmentation driven by phase information and local maximum likelihood
}

Ahror Belaid, Djamal Boukerroui, Yves Maingourd, Jean-Françoise Lerallut

\section{- To cite this version:}

Ahror Belaid, Djamal Boukerroui, Yves Maingourd, Jean-Françoise Lerallut. Implicit active contours for ultrasound images segmentation driven by phase information and local maximum likelihood. 8th International Symposium on Biomedical Imaging (ISBI'11), Mar 2011, Chicago, Illinois, France. pp.630-635. hal-00586401

\section{HAL Id: hal-00586401 https://hal.science/hal-00586401}

Submitted on 15 Apr 2011

HAL is a multi-disciplinary open access archive for the deposit and dissemination of scientific research documents, whether they are published or not. The documents may come from teaching and research institutions in France or abroad, or from public or private research centers.
L'archive ouverte pluridisciplinaire HAL, est destinée au dépôt et à la diffusion de documents scientifiques de niveau recherche, publiés ou non, émanant des établissements d'enseignement et de recherche français ou étrangers, des laboratoires publics ou privés. 


\title{
IMPLICIT ACTIVE CONTOURS FOR ULTRASOUND IMAGES SEGMENTATION DRIVEN BY PHASE INFORMATION AND LOCAL MAXIMUM LIKELIHOOD
}

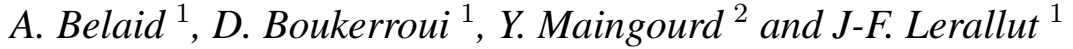 \\ ${ }^{1}$ Heudiasyc, UMR CNRS 6599, Université de Technologie de Compiègne, France \\ ${ }^{2}$ Pediatric Échocardiographic Center, CHU Amiens, France
}

\begin{abstract}
This paper presents a new method in a variational level set framework for ultrasound images segmentation. The conventional intensity gradient based methods have had limited success on ultrasound images. Phase based methods, which are theoretically intensity-invariant, offer a good alternative. The proposed approach uses a speed term based on local phase derived from the monogenic signal. In order to confront more the speckle noise and local changes of intensity, the proposed phase based geodesic active contours term is combined with a new local maximum likelihood region term. A Rayleigh probability distribution is considered to model the B-mode ultrasound images intensities. Preliminary results show that the proposed model is robust to attenuation and captures well the low contrast boundaries.
\end{abstract}

Index Terms - Echocardiography, Level set segmentation, Local phase, Monogenic signal, Maximum likelihood.

\section{INTRODUCTION}

Ultrasound imaging represents one of the most popular exploration technique commonly used in many diagnostic and therapeutic applications. It has many advantages: it is non-invasive, provides images in real time and requires lightweight material. However, ultrasound B-scan images segmentation is particularly difficult mainly due to the low signal-to-noise ratio, low contrast and high amounts of speckle. This image texture, or speckle, is a correlated and multiplicative noise that inherently occurs in all types of coherent imaging systems. Hence, it makes modeling difficult as its statistics depend on the density and on the type of scatterers in the tissues (see e.g., [1]). All these characteristics make segmentation difficult and therefore complicate the diagnosis task [2].

In this study we refer to echocardiographic data. It is known that echocardiography has been one of the driving application areas of medical ultrasound and the literature on

Manuscript received November 11, 2010. This work was supported by a grant of the Regional Council of Picardie and European Union / FEDER.

E-mail:\{ahror.belaid, djamal.boukerroui, jean-francois.lerallut\}@utc.fr, maingourd.yves@chu-amiens.fr. methods for automatically segmenting and tracking the left ventricle is extensive. As it has been pointed in [2], the most popular approach has been to treat echocardiographic endocardial segmentation as a contour finding approach. This is not straightforward as the contrast around the left ventricle chamber boundaries varies, depending on its relative orientation to the transducer direction, and to attenuation. Thus, conventional intensity gradient-based methods have had limited success on typical clinical images. To avoid this drawback, local phase and local image statistics based approaches offer a good alternative, since they make the approach robust to attenuation artifacts. It is within this framework that we propose an alternative in this paper.

Several approaches have been reported in the literature for automated or semiautomated border detection from ultrasound images. For instance, statistical models, arguing that these were more appropriate because of the significant noise and missing boundaries of ultrasound images, have been extensively used [1], [2]. For this reason, several probability density functions were used to model image gray levels statistics $[3,4,5,6]$. Unfortunately, segmentation models driven by global image statistics are not robust to intensity inhomogeneity and, therefore, are not ideal for ultrasound images. More recent techniques attempt to overcome the difficulties caused by intensity inhomogeneities, by using local region information $[7,8,9,10]$.

On the other hand, the local phase based processing has attached a lot of attention in image analysis. Mulet-Parada and Noble [11] were the first to successfully use the local phase information on echocardiographic images. Some phase based level set segmentation methods on medical applications can be found in the literature [12, 13, 14, 15].

This paper concerns the development of a novel segmentation method of the left ventricle within the level set framework. It uses local phase information derived from the monogenic signal $[16,17]$. Our idea is to use a novel speed function, which combines a phase based edge term and a Maximum Likelihood (ML) local region based term. To model gray level behavior of ultrasound images, the classical Rayleigh probability distribution is considered.

In the next section, we describe the monogenic edge detection measure, called Monogenic Feature Asymmetry. The 
proposed segmentation method is presented in Section 3. Section 4 shows preliminary experimental results on real and synthetic data. Section 5 provides a discussion followed by some concluding remarks.

\section{BACKGROUND MATERIALS}

\subsection{Monogenic signal}

One of the popular methods to estimate local signal informations (amplitude, phase and orientation) is based on the analytic representation of this signal. Recently, Felsberg and Sommer [16, 17], proposed a novel n-dimensional generalization of the analytic signal. In particular, they proposed a $2 \mathrm{D}$ isotropic analytic signal, called monogenic signal.

For a given 2D signal $f(\mathbf{x})$, the monogenic signal can be represented by a scalar valued even and vector valued odd filtered responses, with the following simple tick:

$$
\begin{aligned}
\text { even } & =c * f, \\
\text { odd } & =\left(c * h_{1} * f, c * h_{2} * f\right),
\end{aligned}
$$

where $c$ is the spatial domain representation of an isotropic bandpass filter, and $\mathbf{h}=\left(h_{1}, h_{2}\right)$ is the generalized Hilbert transform kernel,

$$
\mathbf{h}(\mathbf{x})=\left(h_{1}, h_{2}\right)=\frac{1}{2 \pi} \frac{\mathbf{x}}{\|\mathbf{x}\|^{3}} .
$$

Boukerroui et al. in $[18,19]$, showed that Cauchy family has better properties. In the frequency domain, a 2D isotropic Cauchy kernel is defined by:

$$
C(\mathbf{u})=n_{c}|\mathbf{u}|^{a} \exp (-s|\mathbf{u}|), a \geq 1,
$$

where $\mathbf{u}=\left(u_{1}, u_{2}\right), s$ is a scaling parameter and $a / s$ is the peak tuning frequency of the filter. $n_{c}$ is a normalization constant, see $[18,19]$ for more details.

\subsection{Monogenic Feature Asymmetry}

Step edge detection is performed using the feature asymmetry measure $(F A)$ of Kovesi [20] defined, in this paper, using the previously presented monogenic signal. The identification of step edges essentially involves finding points where the absolute value of the local phase is $0^{\circ}$ at a positive edge and $180^{\circ}$ at a negative edge. In other words, the difference between the odd and the even filter responses is large. Kovesi suggested to use $F A$ over a number of scales to detect step edge features. We define the multiple scales monogenic feature asymmetry:

$$
F A_{M S}=\frac{1}{N} \sum_{s} \frac{\left\lfloor\left|\operatorname{odd}_{s}\right|-\mid \text { even }_{s} \mid-T_{s}\right\rfloor}{\sqrt{\text { even }_{s}^{2}+\text { odd }_{s}^{2}}+\varepsilon},
$$

where $N$ is the total number of scales, $\lfloor\cdot\rfloor$ denotes zeroing of negative values and $T_{s}$ is the scale specific noise threshold
[20]. For a given scale, the $F A$ takes values in $[0,1]$, close to zero in smooth regions and close to one near boundaries.

The application of Kovesi's $F A$ in [21, 22] on ultrasound images has yielded good results. The authors used steerable filters for the 2D extension. To avoid using steerable filters, the authors in [15] used the monogenic signal, as it is the natural extension of the 1D analytical signal. The monogenic feature asymmetry applied on ultrasound images yielded better results.

\section{SEGMENTATION MODEL}

Let $I$ denote a given image defined on the domain $\Omega$, and let $\mathcal{C}$ be a closed contour represented as the zero level set of a signed distance function $\phi$, i.e., $\mathcal{C}=\{\mathbf{x} \mid \phi(\mathbf{x})=0, \mathbf{x} \in \Omega\}$. We specify the interior of $\mathcal{C}$ by a smooth approximation of the Heaviside function $H(\phi)$. Similarly, the exterior of $\mathcal{C}$ is defined as $(1-H(\phi))$.

We introduce the following classical energy functional to be minimized $[23,5]$ :

$$
\begin{aligned}
E_{G}(\phi) & =\mathcal{L}_{G}(\phi)+\mathcal{R}_{G}(\phi) \\
& =\lambda \int_{\Omega} g \delta(\phi)|\nabla \phi| d \mathbf{x} \\
& -\int_{\Omega} H(\phi) \log p(I) d \mathbf{x}-\int_{\Omega}(1-H(\phi)) \log p(I) d \mathbf{x} .
\end{aligned}
$$

This energy model is composed of two terms: a gradient edge based term $\mathcal{L}_{G}(\phi)$ and a global region based term $\mathcal{R}_{G}(\phi)$. The first term is the Geodesic Active Contour term (GAC) [24], were $\lambda$ is a positive fixed parameter and $g$ is an inverse edge indicator function, generally taken as $g(x, y)=$ $1 /\left(1+\left|\nabla G_{\sigma} * I\right|\right)$. Here, $G_{\sigma}$ is the Gaussian kernel with standard deviation $\sigma$. The second term is a global region based term. Specifically, it is the log likelihood function to maximize, given by the product of the inner and the outer probabilities [25, 23]. Here, $p(I)$ represents the probability density function characterizing the observed gray level of image $I$.

In the following, we define an alternative energy function, similar to the form of (2), but using local image properties. We use a local phase based edge indicator function $g=1 /\left(1+\gamma F A_{M S}\right)$ instead of the classical inverse gradient based one. Here, $\gamma$ is a scale parameter and $F A_{M S} \in[0,1]$ represents the monogenic feature asymmetry measure defined by (1). This allows us to define the local phase based GAC term noted $\mathcal{L}_{P}(\phi)$. As it was mentioned in the introduction, recent works showed that ultrasound images respond well to phase based edge detection. Moreover, a multi-scales approach offers a better control on the edge detection quality.

Now, we focus on our new local region term. Classical region based methods, like the ML model, often make strong assumptions on the intensity distributions of the searched object and background. In order to be less restrictive, the local energy achieves a trade off between local features and global 
region. Furthermore, the localization seems to be a good alternative to avoid the attenuation artifact, which is one of the main characteristics of ultrasound images. We aim in the remainder of this section to change this term $\mathcal{R}_{G}$ to a new local region based term $\mathcal{R}_{L}[26,7,8]$. This term is a local version of the one presented by Sarti and al. in [5]. Thus, we use the Rayleigh probability distribution $p(I)=I / \sigma^{2} \exp \left(-I^{2} / 2 \sigma^{2}\right)$, to model the behavior of the observed gray levels.

To achieve this, we introduce the following characteristic function $\mathcal{B}$ used to define a local region in terms of a radius parameter $r$ [7],

$$
\mathcal{B}(\mathbf{y} ; \mathbf{x})= \begin{cases}1, & \|\mathbf{x}-\mathbf{y}\|<r \\ 0, & \text { otherwise } .\end{cases}
$$

Thus, the local region version around a given point $\mathbf{x}$, of the global region term $\mathcal{R}_{G}$ in (2), is given by

$$
\begin{aligned}
F(\phi ; \mathbf{x})= & -\int_{\Omega} \mathcal{B}(\mathbf{y} ; \mathbf{x}) H(\phi) \log p(I) d \mathbf{y} \\
& -\int_{\Omega} \mathcal{B}(\mathbf{y} ; \mathbf{x})(1-H(\phi)) \log p(I) d \mathbf{y},
\end{aligned}
$$

this formulation allow as to estimate the $p d f$ parameter $\sigma^{2}$ locally, inside and outside the curve. The local ML estimates are given by:

$$
\begin{aligned}
\widehat{\sigma}_{i}^{2}(\mathbf{x}) & =\frac{1}{2 M_{i}(\mathbf{x})} \int_{\Omega} \mathcal{B}(\mathbf{y} ; \mathbf{x}) H(\phi) I^{2} d \mathbf{y} \\
\widehat{\sigma}_{o}^{2}(\mathbf{x}) & =\frac{1}{2 M_{o}(\mathbf{x})} \int_{\Omega} \mathcal{B}(\mathbf{y} ; \mathbf{x})(1-H(\phi)) I^{2} d \mathbf{y}
\end{aligned}
$$

were $M i$ and $M_{o}$ denote respectively the local area inside and outside $\Omega$ and are given as:

$$
\begin{aligned}
M_{i}(\mathbf{x}) & =\int_{\Omega} \mathcal{B}(\mathbf{y} ; \mathbf{x}) H(\phi) d \mathbf{y} \\
M_{e}(\mathbf{x}) & =\int_{\Omega} \mathcal{B}(\mathbf{y} ; \mathbf{x})(1-H(\phi)) d \mathbf{y} .
\end{aligned}
$$

By introducing these estimates back in the local loglikelihood (3), we obtain the new formulation:

$$
\begin{aligned}
& F(\phi ; \mathbf{x})= \\
& \quad-M_{i}(\mathbf{x}) \log \left(\frac{1}{M_{i}(\mathbf{x})} \int_{\Omega} \mathcal{B}(\mathbf{y} ; \mathbf{x}) H(\phi) I^{2} d \mathbf{y}\right) \\
& \quad-M_{o}(\mathbf{x}) \log \left(\frac{1}{M_{o}(\mathbf{x})} \int_{\Omega} \mathcal{B}(\mathbf{y} ; \mathbf{x})(1-H(\phi)) I^{2} d \mathbf{y}\right),
\end{aligned}
$$

By bringing the local phase and local region terms, we now define our new energy built from (2) as follow:

$$
\begin{aligned}
E_{L}(\phi) & =\mathcal{L}_{P}(\phi)+\mathcal{R}_{L}(\phi) \\
& =\lambda \int_{\Omega} g \delta(\phi)|\nabla \phi| d \mathbf{y}+\int_{\Omega} \delta(\phi(\mathbf{x})) F(\phi ; \mathbf{x}) d \mathbf{x}
\end{aligned}
$$

$F(. ; \mathbf{x})$ represents a local image contribution used at each point along the contour to evolve this contour.

This formulation is under the assumption that the local behavior of an ultrasound image follows the Rayleigh distribution, and assuming that the size of this local region is sufficient for a maximum likelihood estimation of the parameter $\sigma^{2}$.

It is straightforward to see that the mask $\mathcal{B}(\mathbf{y} ; \mathbf{x})$ is independent of $\phi$. Thus, the associated flow equation of $F(\phi ; \mathbf{x})$ is given by:

$$
\begin{gathered}
F_{L M L}(\phi ; \mathbf{z} ; \mathbf{x})=\log \left(\frac{1}{M_{i}(\mathbf{x})} \int_{\Omega} \mathcal{B}(\mathbf{y} ; \mathbf{x}) H(\phi(\mathbf{y})) I(\mathbf{y})^{2} d \mathbf{y}\right) \\
+\frac{I(\mathbf{z})^{2} M_{i}(\mathbf{x})+\int_{\Omega} \mathcal{B}(\mathbf{y} ; \mathbf{x}) H(\phi(\mathbf{x})) I(\mathbf{y})^{2} d \mathbf{y}}{\int_{\Omega} \mathcal{B}(\mathbf{y} ; \mathbf{x}) H(\phi(\mathbf{x})) I(\mathbf{y})^{2} d \mathbf{y}} \\
-\log \left(\frac{1}{M_{e}(\mathbf{x})} \int_{\Omega} \mathcal{B}(\mathbf{y} ; \mathbf{x})(1-H(\phi(\mathbf{y}))) I(\mathbf{x})^{2} d \mathbf{y}\right) \\
+\frac{I(\mathbf{z})^{2} M_{e}(\mathbf{x})+\int_{\Omega} \mathcal{B}(\mathbf{y} ; \mathbf{x})(1-H(\phi(\mathbf{y}))) I(\mathbf{y})^{2} d \mathbf{y}}{\int_{\Omega} \mathcal{B}(\mathbf{y} ; \mathbf{x})(1-H(\phi(\mathbf{y}))) I(\mathbf{y})^{2} d \mathbf{y}},
\end{gathered}
$$

in agreement with [5].

Finally, the gradient descent flow minimizing (4), in the level set formulation, is given by (see Appendix):

$$
\begin{aligned}
\frac{\partial \phi}{\partial \tau}(\mathbf{x}) & =\delta(\phi(\mathbf{x}))\left[\lambda \operatorname{div}\left(g(\mathbf{x}) \frac{\nabla \phi(\mathbf{x})}{|\nabla \phi(\mathbf{x})|}\right)\right. \\
& \left.+\int_{\Omega} \delta(\phi(\mathbf{y})) \mathcal{B}(\mathbf{y} ; \mathbf{x}) F_{L M L}(\phi ; \mathbf{y} ; \mathbf{x}) d \mathbf{y}\right]
\end{aligned}
$$

\section{RESULTS}

As a preliminary validation, we have compared the semiautomatic algorithm results to manual segmentations. We have collected a set of 10 bidimensional cardiac ultrasound images for different patients, obtained from a Philips IE33 echocardiographic imaging system. The data set was segmented by two specialists in an independent way, i.e. in different days, at the Pediatric Echocardiographic Center, CHU Amiens. Each specialist segments each image 5 times, so that 10 manual segmentations are available for each image. Thus, in all, we have 100 manual segmentations.

Matlab 7.6 (R2008a) was used for the implementation of the proposed method. In all of the experimental results, the following parameters were fixed as such : bandwidth $2.5 \mathrm{oc}-$ taves as suggested in [18], wavelength $=[20,22,24,26]$ pixels. The radius of the localizing ball $r$ was fixed to 11 pixels. Unless otherwise stated, $\lambda$ was set to 1 .

The experiment in Fig. 1 shows the performance of the monogenic feature asymmetry to detect step edge boundaries in very noisy and low contrast data. The adverse effect of FA measure is the delocalization, by moving closer to finer 

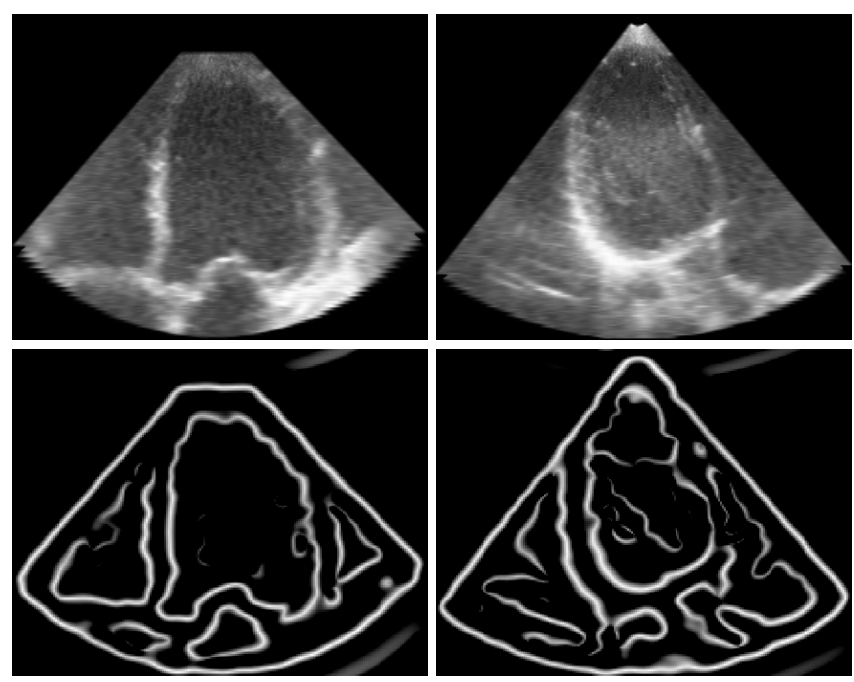

Fig. 1. Example of monogenic feature asymmetry at a given scale $s>0$. Top: original images, bottom: edge detections.
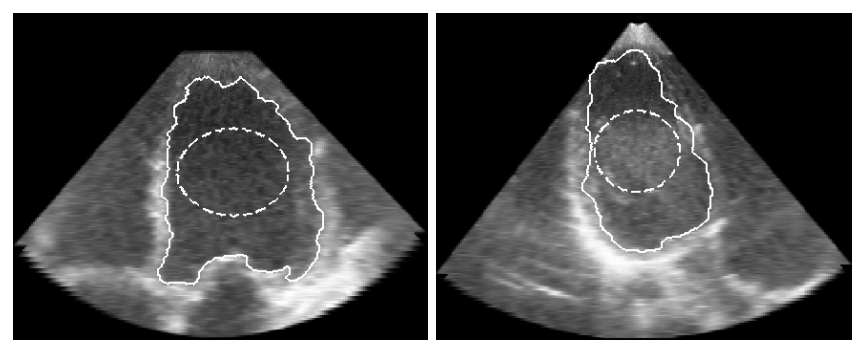

Fig. 2. Example of the proposed model segmentation results of the left ventricle. The inner dashed contours are the respective initializations. In this experiments, $\lambda=0.7$.

Table 1. Performance indices measures DSC and MAD versus Interobserver, Intraobserver and computer-observer distances. The mean, median and standard deviation for DSC and MAD are shown.

\begin{tabular}{|l|ccc|ccc|}
\hline Measures & \multicolumn{3}{|c|}{ DSC (\%) } & \multicolumn{3}{c|}{ MAD (pixels) } \\
\hline \hline Indexes & Mean & Median & SD & Mean & Median & SD \\
\hline \hline GAC & 87.30 & 88.65 & 3.13 & 5.25 & 4.96 & 1.57 \\
GAC+GML & 89.33 & 89.58 & 3.31 & 4.43 & 4.17 & 1.66 \\
LP+LML & 91.90 & 92.70 & 2.53 & 3.53 & 3.17 & 1.48 \\
\hline \hline Interobserver & 94.82 & 95.51 & 2.75 & 2.42 & 2.65 & 0.94 \\
Intraobserver & 96.10 & 96.54 & 0.83 & 1.93 & 1.84 & 0.27 \\
\hline
\end{tabular}

scales, the FA measure recovers details and discontinuities, but looses regularity and continuity of the boundaries. This drawback can be reduced by using a multiscale detection, see equation (1).

Fig. 2 shows illustrative results of our method on two typical ultrasound images (left ventricle). Fig. 3 shows illustrative comparison results of the proposed segmentation algorithm and the results of the global ML algorithm [5] with manual delineation. These results give the reader some insight re-
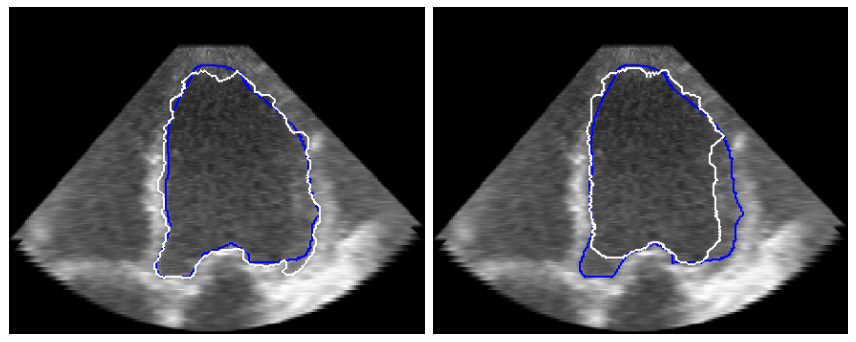

Fig. 3. Comparison of the proposed method (left) and the global ML model (right) with a manual delineation. Blue line: manual delineation, white line: semi automatic segmentation. Parameter $\lambda=0.7$.
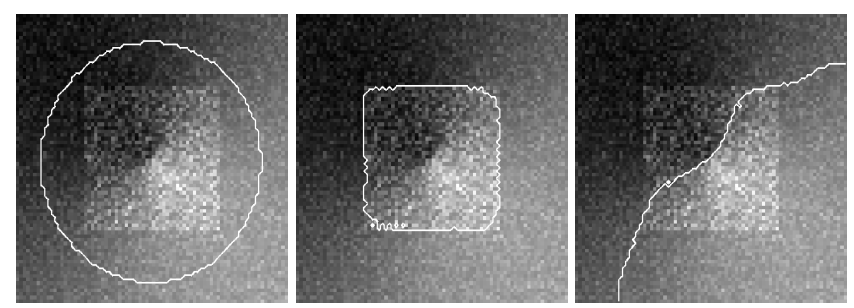

Fig. 4. Comparison of the local and global ML model. Left to right: Initial contour and original synthetic image, segmentation using respectively local and global ML region term only.

garding the robustness to speckle noise and to attenuation.

The last example, Fig. 4 demonstrates the effect of a nonuniform illumination with Speckle noise. The edge based term is turned off, wile the region term play the leading role. In this case, the local region based term gives a successful result while the global region based segmentation algorithm fails.

Table 1 shows a quantitative comparison between our approach -noted LP+LML for local phase with a local ML model- and two semi-automatic segmentation methods : classical GAC and GAC with a global ML model (GAC+GML). Inter and Intra-observer values are also shown in this table. The mean, median and standard deviation of all the echocardiographic images segmentations are shown for both measures: Dice similarity coefficient (DSC) and mean absolute distance (MAD) (See, for example, [27, 6, 15]).

\section{DISCUSSION AND CONCLUSION}

We have presented a new approach of ultrasound images segmentation. We use known techniques: geodesic active contours and Maximum Likelihood approach. The combination takes advantage of the benefits of both techniques.

Ultrasound images characteristics, such as attenuation and low contrast, suggest the use of local image properties in order to improve robustness and accuracy. In our approach, the local phase based GAC term was reinforced by a local region based term in order to improve the capture range and the 
sensitivity of the algorithm to local minima, see Fig.3. Local model parameter estimation is necessary to preserve the robustness of the algorithm to attenuation.

The radius parameter $r$ is very important for the segmentation process. It can be adjusted according to the image intensity inhomogeneities. It is advisable not to use a very small value of $r$ to ovoid a high variance in parameter estimation, nor a very large value to avoid a high bias. This parameter must be relatively small and sufficiently large. We also note that the localization adverse effect of FA discussed in the previous section can be corrected by the local region term.

The qualitative evaluation on the natural and synthetic data shows, as expected, that the use of phase based edge detection with an additional local region term provides a significant improvement with respect to the classical GAC and GAC+GML, see Table 1. A key advantage of this approach is that it is more robust to intensity inhomogeneities, as it is demonstrated in Fig.4. Although only preliminary results are shown, our experiments suggest that the proposed terms can favorably outperform classical ones of the same nature.

\section{Acknowledgment}

The authors thank the Regional Council of Picardie and European Union / FEDER for their financial support, and Dr. Mathiron and Dr. Levy for their help in the clinical evaluation of the data.

\section{A. FUNCTIONAL MINIMIZATION}

To compute the first variation of equation (3), we express $F(\phi ; \mathbf{x})$ as $F(\phi+\tau \psi ; \mathbf{x})$ and take the partial derivative with respect to $\tau$ evaluated at $\tau=0$. This allows us to represent a tiny differential of movement. Here, $\psi$ represents a small perturbation along the normal direction of $\phi$ weighted by a scalar $\tau$. In agreement to [5], we obtain

$$
\begin{aligned}
\left.\frac{\partial}{\partial \tau} F(\phi+\tau \psi ; \mathbf{x})\right|_{\tau=0}= & \int_{\Omega} \delta(\phi(\mathbf{y})) \mathcal{B}(\mathbf{y} ; \mathbf{x}) \\
& \times F_{L M L}(\phi ; \mathbf{y} ; \mathbf{x}) \psi(\mathbf{y}) d \mathbf{y},
\end{aligned}
$$

were $F_{L M L}($.$) is the associated flow equation of F($.$) as ex-$ pressed in (5).

Moreover, starting from the local energy term $\mathcal{R}_{L}(\phi)$ given in (4),

$$
\mathcal{R}_{L}(\phi)=\int_{\Omega} \delta(\phi(\mathbf{x})) F(\phi ; \mathbf{x}) d \mathbf{x},
$$

the tiny differential of movement is given by

$$
\begin{aligned}
\left.\frac{\partial}{\partial \tau} \mathcal{R}_{L}(\phi+\tau \psi)\right|_{\tau=0}= & \frac{\partial}{\partial \tau}\left\{\int_{\Omega} \delta(\phi(\mathbf{x})+\tau \psi(\mathbf{x}))\right. \\
& \times F(\phi+\tau \psi ; \mathbf{x}) d \mathbf{x}\}\left.\right|_{\tau=0},
\end{aligned}
$$

by the product rule, we obtain the following:

$$
\begin{aligned}
d \mathcal{R}_{L}(\phi, \psi)= & \int_{\Omega} \delta^{\prime}(\phi(\mathbf{x})) \psi(\mathbf{x}) F(\phi ; \mathbf{x}) d \mathbf{x} \\
& +\int_{\Omega} \delta(\phi(\mathbf{x})) d F(\phi, \psi ; \mathbf{x}) d \mathbf{x}
\end{aligned}
$$

were $d F(\phi, \psi ; \mathbf{x})$ denotes the directional derivative of $F(\phi ; \mathbf{x})$ as presented in the equation (6). $\delta^{\prime}(\phi)$ denotes the derivative of $\delta(\phi)$. This term is ignored, because it does not affect the movement of the curve [7]. The equation of $d \mathcal{R}_{L}(\phi, \psi)$ becomes:

$$
\begin{aligned}
d \mathcal{R}_{L}(\phi, \psi) & =\int_{\Omega} \delta(\phi(\mathbf{x})) \\
\times & \left\{\int_{\Omega} \delta(\phi(\mathbf{y})) \mathcal{B}(\mathbf{y} ; \mathbf{x}) F_{L M L}(\phi ; \mathbf{y} ; \mathbf{x}) \psi(\mathbf{y}) d \mathbf{y}\right\} d \mathbf{x} .
\end{aligned}
$$

By moving the integral over $\mathbf{y}$ outside the integral over $\mathbf{x}$ we obtain:

$$
\begin{aligned}
& d \mathcal{R}_{L}(\phi, \psi)=\int_{\Omega} \int_{\Omega} \delta(\phi(\mathbf{x})) \delta(\phi(\mathbf{y})) \\
& \quad \times \mathcal{B}(\mathbf{y} ; \mathbf{x}) F_{L M L}(\phi ; \mathbf{y} ; \mathbf{x}) \psi(\mathbf{y}) d \mathbf{x} d \mathbf{y}
\end{aligned}
$$

From this equation it is easy to determine the associated flow:

$$
\frac{\partial \phi}{\partial \tau}(\mathbf{x})=\delta(\phi(\mathbf{x})) \int_{\Omega} \delta(\phi(\mathbf{y})) \mathcal{B}(\mathbf{y} ; \mathbf{x}) F_{L M L}(\phi ; \mathbf{y} ; \mathbf{x}) d \mathbf{y}
$$

\section{B. REFERENCES}

[1] S. Nadarajah, "Statistical distributions of potential interest in ultrasound speckle analysis," Phys. Med. Biol., vol. 52, pp. 213-227, 2007.

[2] J. A. Noble and D. Boukerroui, "Ultrasound image segmentation: A survey," IEEE Trans. Med. Imag., vol. 25, no. 8, pp. 987-1010, 2006.

[3] M. Mignotte, J. Meunier, and J.-C. Tardif, "Endocardial boundary estimation and tracking in echocardiographic images using deformable template and markov random fields," Pattern Anal. \& Appl., vol. 4, no. 4, pp. 256-271, 2001.

[4] D. Boukerroui, A. Baskurt, J. A. Noble, and O. Basset, "Segmentation of ultrasound images: multiresolution 2D and 3D algorithm based on global and local statistics," Pattern Recogn. Lett., vol. 24, no. 4-5, pp. 779-790, 2003. 
[5] A. Sarti, C. Corsi, E. Mazzini, and C. Lamberti, "Maximum likelihood segmentation of ultrasound images with rayleigh distribution," IEEE Trans. Ultra. Fer. Freq. Control, vol. 52, no. 6, pp. 947-960, June 2005.

[6] Y. Zhu, X. Papademetris, A. J. Sinusas, and J. S. Duncan, "A coupled deformable model for tracking myocardial borders from real-time echocardiography using an incompressibility constraint," Med. Image Anal., vol. 14, no. 3, 2010.

[7] S. Lankton and A. Tannenbaum, "Localizing regionbased active contours," IEEE Trans. Image Process., vol. 17, no. 11, pp. 2029-2039, 2008.

[8] T. Brox and D. Cremers, "On local region models and a statistical interpretation of the piecewise smooth mumford-shah functional," Int. J. Comp. Vision, vol. 84, no. 2, pp. 184-193, 2009.

[9] C. Li, C. Kao, J. Gore, and Z. Ding, "Minimization of region-scalable fitting energy for image segmentation," IEEE Trans. Image Process., vol. 17, no. 10, pp. 19401949, 2008.

[10] M. Alessandrini, T. Dietenbeck, D. Barbosa, J. D’Hooge, O. Basset, N. Speciale, D. Friboulet, and O. Bernard, "Segmentation of the full myocardium in echocardiography using constrained level-sets." in Comput. Cardiol., Belfast, Northern Ireland, United Kingdom, Sept. 2010.

[11] M. Mulet-Parada and J. A. Noble, "2D+T acoustic boundary detection in echocardiography," Med. Image Anal., vol. 4, no. 1, pp. 21 - 30, 2000.

[12] I. Hacihaliloglu, R. Abugharbieh, A. Hodgson, and R. Rohling, "Bone segmentation and fracture detection in ultrasound using 3D local phase features," in $M I C$ CAI. Berlin, Heidelberg: Springer-Verlag, 2008, pp. 287-295.

[13] G. Läthén, J. Jonasson, and M. Borga, "Phase based level set segmentation of blood vessels," in Proc. of 19th ICPR. Tampa, FL, USA: IAPR, Dec. 2008, pp. 1-4.

[14] R. Ali, M. J. Gooding, M. Christlieb, and J. M. Brady, "Phase-based segmenation of cells with brightfield microscopy," in IEEE Symposium on Biomedical Imaging, 2007, pp. 57-60.

[15] A. Belaid, D. Boukerroui, Y. Maingourd, and J.-F. Lerallut, "Phase based level set segmentation of ultrasound images," IEEE Trans. Information Tech. Biomed., vol. 15, no. 1, pp. 138-147, 2011.

[16] M. Felsberg and G. Sommer, "The monogenic signal," IEEE Trans. Signal Process., vol. 49, no. 49, pp. 31363144, 2001.
[17] — - "The monogenic scale-space: A unifying approach to phase-based image processing in scale-space," J. Math. Imag. Vision, vol. 21, no. 1, pp. 5-26, 2004.

[18] D. Boukerroui, J. A. Noble, and M. Brady, "On the choice of band-pass quadrature filters," J. Math. Imag. Vision, vol. 21, no. 1, pp. 53-80, 2004.

[19] _ Frontiers in Robotics Research, Max A. Denket ed. Nova Publishers, 2006, ch. On the Selection of Band-Pass Quadrature Filters, pp. 67-111.

[20] P. Kovesi, "Image features from phase congruency," Videre: J. Comput. Vis. Res., vol. 1, no. 3, pp. 1-26, 1999.

[21] M. Mulet-Parada and J. A. Noble, "2D+T acoustic boundary detection in echocardiography," in MICCAI. London, UK: Springer-Verlag, 1998, pp. 806-813.

[22] D. Boukerroui, J. A. Noble, M. C. Robini, and J. Brady, "Enhancement of contrast regions in sub-optimal ultrasound images with application to echocardiography," Ultrasound Med. Biol, vol. 27, no. 12, pp. 1583-1594, 2001.

[23] V. B. C. Chesnaud, P. Refregier, "Statistical region snake-based segmentation adapted to different physical noise models," IEEE Trans. Pattern Anal. Mach. Intell., vol. 21, no. 11, pp. 1145-1157, 1999.

[24] V. Caselles, R. Kimmel, and G. Sapiro, "Geodesic active contours," Int. J. Comp. Vision, vol. 22, no. 1, pp. 61-79, 1997.

[25] A. C. Bovik, "On detecting edges in speckle imagery," IEEE Trans. Acoust. Speech Signal Processing, vol. 36, no. 10, pp. 1618-1627, 1988.

[26] C. Li, C. Kao, J. Gore, and Z. Ding, "Implicit active contours driven by local binary fitting energy," in Proc. of IEEE Conf. Comput. Vis. Pattern Recognit. (CVPR). Washington, DC, USA: IEEE Computer Society, 2007, pp. 1-7.

[27] V. Chalana, D. T. Linker, D. R. Haynor, and Y. Kim, “A multiple active contour model for cardiac boundary detection on echocardiographic sequences," IEEE Trans. Med. Imaging, vol. 15, no. 3, pp. 290-298, June 1996. 